\title{
Mevalonate Pathway-mediated ER Homeostasis Is Required for Haploid Stability in Human Somatic Cells
}

\author{
Kan Yaguchi ${ }^{1}$, Kimino Sato ${ }^{1}$, Koya Yoshizawa ${ }^{1}$, Daisuke Mikami², Kohei Yuyama ${ }^{2}$, \\ Yasuyuki Igarashi ${ }^{2}$, Gabor Banhegyi ${ }^{3}$, Eva Margittai ${ }^{4}$, and Ryota Uehara ${ }^{1,5^{*}}$ \\ ${ }^{1}$ Graduate School of Life Science, Hokkaido University, Japan, ${ }^{2}$ Lipid Biofunction Section, Faculty of \\ Advanced Life Science, Hokkaido University, Japan, ${ }^{3}$ Institute of Biochemistry and Molecular Biology, \\ Semmelweis University, Hungary, ${ }^{4}$ Institute of Translational Medicine, Semmelweis University, Hungary, \\ ${ }^{5}$ Faculty of Advanced Life Science, Hokkaido University, Japan
}

\begin{abstract}
The somatic haploidy is unstable in diplontic animals, but cellular processes determining haploid stability remain elusive. Here, we found that inhibition of mevalonate pathway by pitavastatin, a widely used cholesterol-lowering drug, drastically destabilized the haploid state in HAP1 cells. Interestingly, cholesterol supplementation did not restore haploid stability in pitavastatin-treated cells, and cholesterol inhibitor U18666A did not phenocopy haploid destabilization. These results ruled out the involvement of cholesterol in haploid stability. Besides cholesterol perturbation, pitavastatin induced endoplasmic reticulum (ER) stress, the suppression of which by a chemical chaperon significantly restored haploid stability in pitavastatin-treated cells. Our data demonstrate the involvement of the mevalonate pathway in the stability of the haploid state in human somatic cells through managing ER stress, highlighting a novel link between ploidy and ER homeostatic control.
\end{abstract}

Key words: haploid, ER stress, Mevalonate pathway

\section{Introduction}

In diplontic animal organisms, somatic haploidy is generally unstable, causing frequent autodiploidization at the cellular level and severe developmental abnormalities at the organismal level (Sagi and Benvenisty, 2017; Wutz, 2014). The halving of genome copy from the normal diploid state potentially has pleiotropic effects on cellular homeostasis in haploid cells. An apparent feature of haploid cells is their halved cellular volume to diploids with the halving of total protein content (Yaguchi et al., 2018). Though these features possibly have profound influence on intracellular processes - such as the metabolic control-in haploid state, it remains elusive what aspects of the metabolism alter and characterize cellular phenotypes of haploid cells.

The mevalonate pathway metabolizes acetyl-CoA to produce sterol isoprenoids, and non-sterol isoprenoids that mediate diverse biosynthetic processes essential for cell construction and proliferation (Buhaescu and Izzedine, 2007; Mullen et al., 2016). Among mevalonate-derived

\footnotetext{
*To whom correspondence should be addressed: Ryota Uehara, Faculty of Advanced Life Science, Hokkaido University, Kita 21, Nishi 11, Kita-ku, Sapporo 001-0021, Japan.

Tel: +81-11-706-9238

E-mail: ruehara@sci.hokudai.ac.jp
}

metabolites, cholesterol serves as a structural component of cell membranes and a precursor of fundamental biomolecules, such as steroid hormones. Mevalonate-derived polyisoprenols, such as dolichol phosphates are essential components of glycoprotein synthesis and endoplasmic reticulum (ER) homeostasis participating in protein Nglycosylation, C- and O-mannosylation, and GPI-anchor production (Carlberg et al., 1996; Chojnacki and Dallner, 1988; Doucey et al., 1998). Mevalonate-derived isoprenoids are also used for the prenylation of small GTPases, which mediates signal transduction for dynamic processes such as cytoskeletal reorganization and vesicular trafficking (Leung et al., 2006; Wang and Casey, 2016). Mevalonate pathway controls cell size by optimizing mitochondrial functionality through protein prenylation (Miettinen and Björklund, 2015, 2016; Miettinen et al., 2014). Inhibition of the rate-limiting enzyme of mevalonate pathway, 3hydroxy-3-methylglutaryl-coenzyme A reductase (HMGCR) by statins perturbs this homeostatic control, leading to increased cell size in cultured cells (Miettinen and Björklund, 2015).

In this study, in an attempt to modulate cell size by an HMGCR inhibitor pitavastatin in human haploid HAP1 cells (Carette et al., 2011), we found that the inhibitor compromised the stability of the haploid state in these cells. 
Interestingly, a recent chemical screen searching for compounds that stabilize haploid state has also identified statins leading to the selective loss of haploid cells (Olbrich et al., 2019). However, whether the mevalonate pathway is indeed involved in promoting haploid stability, and how the inhibition of the pathway may lead to destabilization of haploid state is still unknown. Using a pharmacological approach, we specified that statin-induced ER stress as a process responsible for the destabilization of the haploid state.

\section{Materials and Methods}

\section{Cell culture and flow cytometry}

HAP1 cells were purchased from Haplogen and cultured in Iscove's Modified Dulbecco's Medium (IMDM; Wako) supplemented with $10 \%$ fetal bovine serum and $1 \times$ antibiotic-antimycotic (Sigma-Aldrich). Haploid cells were regularly maintained by sizebased cell sorting, as previously described (Yaguchi et al., 2018). For DNA content analysis, cells were stained with $10 \mu \mathrm{g} / \mathrm{ml}$ Hoechst 33342 (Dojindo) for $15 \mathrm{~min}$ at $37^{\circ} \mathrm{C}$, and fluorescence intensity was analyzed using a JSAN desktop cell sorter (Bay bioscience). For the long-term passage experiments, freshly purified haploid cells were cultured, with passage typically once two days, in the presence of different compounds at final concentrations described elsewhere.

\section{Cholesterol measurement}

Details on the cholesterol measurement are described in Supplemental Materials and methods.

\section{Chemical compounds}

Compounds were purchased from suppliers as follows: Pitavastatin (163-24861, Wako); U18666A (10009085, Cayman Chemical); Cholesterol (SLBZ0657, Sigma-Aldrich); FTI-277 (S7465, Selleck); GGTI-298 (S7466, Selleck); tauroursodeoxycholic acid (TUDCA, T1567, Tokyo Chemical Industry); and mevalonate (mevalonolactone, M4667, Sigma-Aldrich).

\section{Antibodies and immunoblotting}

Antibodies used in this study are listed in Table S1. Phos-tag SDS-PAGE for PERK immunoblotting was conducted as previously described (Yang et al., 2010). We used the ezWestLumi plus ECL Substrate (ATTO) and a LuminoGraph II chemiluminescent imaging system (ATTO) for immunoblotting signal detection. We used the Gels tool in ImageJ (NIH) for signal quantification.

\section{Results}

\section{Mevalonate production is required for the stability of the haploid state in HAP1 cells}

To test the effects of inhibition of the mevalonate pathway on cell size control, we treated human haploid cell HAP1 with a competitive HMGCR inhibitor pitavastatin, which has been reported to increase the size of human cell lines such as Jurkat (Miettinen and Björklund, 2015). Although we did not observe the increase of cell size in HAP1 cells upon pitavastatin treatment (Fig. S1), we unexpectedly found that the inhibitor destabilized the haploid state of HAP1 cells in flow cytometric DNA content analysis (Fig. 1A-D). Freshly purified, non-treated haploid HAP1 cells gradually diploidized during $\sim 3$-weeks continuous passages, resulting in the reduction in haploid $\mathrm{G} 1$ proportion (1C peak) from $37.4 \pm 1.0 \%$ to $28.0 \pm 1.7 \%$ (mean \pm standard error, $\mathrm{n}=3$; Fig. 1A-D) (Yaguchi et al., 2018). Diploid $\mathrm{G} 2 / \mathrm{M}$ proportion (4C peak) concomitantly increased during the continuous passages from $1.5 \pm 0.4 \%$ to $10.4 \pm 1.5 \%$ (Fig. $1 \mathrm{~A}-\mathrm{D})$. However, when treated with $0.5 \mu \mathrm{M}$ pitavastatin, which allowed long-term culture without blocking cell proliferation, haploid G1 proportion became significantly less than non-treated control (17.1 $\pm 1.2 \%$; Fig. 1C and D). Diploid G2/M proportion concomitantly became substantially more in pitavastatin-treated culture than in non-treated control (18.2 $\pm 1.3 \%$; Fig. 1C and D). Since this result indicates the importance of statin-targeted processes in haploid stability, we further investigated the mechanism underlying the statin-mediated destabilization of haploid state. First, we tested whether co-treatment with mevalonate ameliorates haploid stability in the presence of pitavastatin (Fig. $1 \mathrm{C}$ and $\mathrm{D})$. Mevalonate supplementation significantly preserved the haploid population in pitavastatin-treated culture (haploid G1 or diploid G2/M proportion of $30.2 \pm 1.0 \%$ or $9.4 \pm 0.9 \%$, respectively; Fig. $1 \mathrm{C}$ and D), demonstrating the requirement for a sufficient amount of mevalonate for haploid stability in HAP1 cells.

\section{Cholesterol perturbation is not the cause of haploid destabilization by pitavastatin}

Next, we determined downstream branches of the mevalonate pathway crucial for the maintenance of haploid state. Because statins are widely used cholesterol-lowering drugs (Adhyaru and Jacobson, 2018), we addressed the possible involvement of the cholesterol branch in haploid stability. For this, we compared the content of total cholesterol extracted from control and pitavastatin-treated HAP1 cells using a colorimetric method (Fig. 2A; Materials and methods). In this assay, we did not observe a significant difference in cholesterol content between control and pitavastatin-treated cells. This suggests that, at such a low concentration as $0.5 \mu \mathrm{M}$, pitavastatin does not drastically 

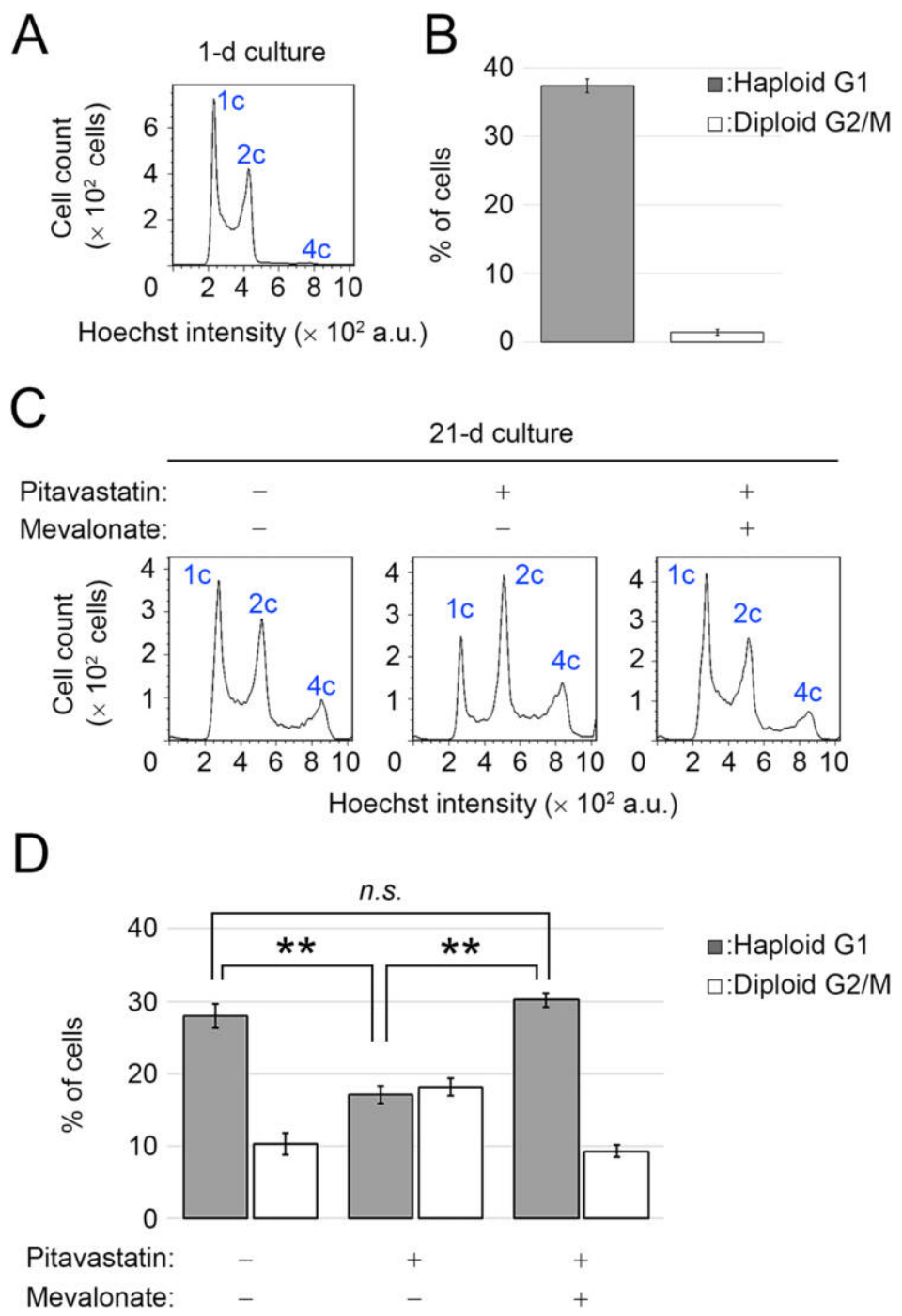

Fig. 1. Destabilization of the haploid state by inhibition of the mevalonate pathway in HAP1 cells. (A, C) Flow cytometric analysis of DNA content in Hoechst-stained cells. Cells were analyzed one day after cell thawing (A), or after $21-\mathrm{d}$ culture in the absence or presence of $0.5 \mu \mathrm{M}$ pitavastatin with or without $20 \mu \mathrm{M}$ mevalonate supplementation (C). (B, D) The proportion of the haploid G1 (1 c peak) or diploid G2/M (4 c peak) population in A or C. Means \pm standard error (SE) of three independent experiments (day 1 in B and day 20 or 21 in D, ** $p<0.01$, n.s.: not significant, one-way ANOVA with Tukey post-hoc test).

block cholesterol synthesis. We next visualized intracellular distribution and the content of cholesterol at the single-cell level using a cholesterol-binding fluorescent compound filipin (Fig. 2B) (Drabikowski et al., 1973). In control cells, filipin fluorescence signal distributed throughout the plasma- and intracellular membrane structures, and $0.5 \mu \mathrm{M}$ pitavastatin modestly reduced the filipin staining intensity (Fig. 2B-D). Next, we addressed whether cholesterol supplementation is sufficient to restore haploid stability in pitavastatin-treated cells. The addition of $10 \mu \mathrm{M}$ cholesterol to the pitavastatin-treated cell culture fully restored cholesterol level (Fig. 2A-D). However, cholesterol supplementation did not change haploid G1 or diploid G2/M proportion in statin-treated culture (Fig. 2E and F). On the other hand, mevalonate supplementation, which fully restored haploid stability in statin-treated cells (Fig. 1), did not increase the cholesterol level in statin-treated cells (Fig. 2A and D). These data demonstrated that lowered cholesterol level was not the cause of haploid destabilization by pitavastatin.

Next, we tested the effect of perturbation of cholesterol homeostasis by a non-statin cholesterol inhibitor on haploid stability. An amphipathic steroid U18666A perturbs the cholesterol-mediated bioprocesses by inhibiting both synthesis and intracellular transport of cholesterol (Cenedella, 2009). Treatment with $2.5 \mu \mathrm{M}$ U18666A resulted in the accumulation of cholesterol in intracellular vesicles, a typi- 
A

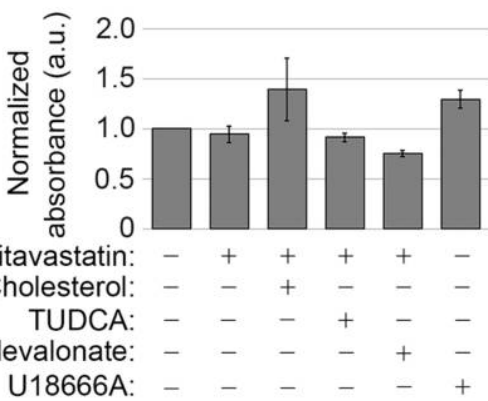

B

U18666: $-\quad-\quad-+$

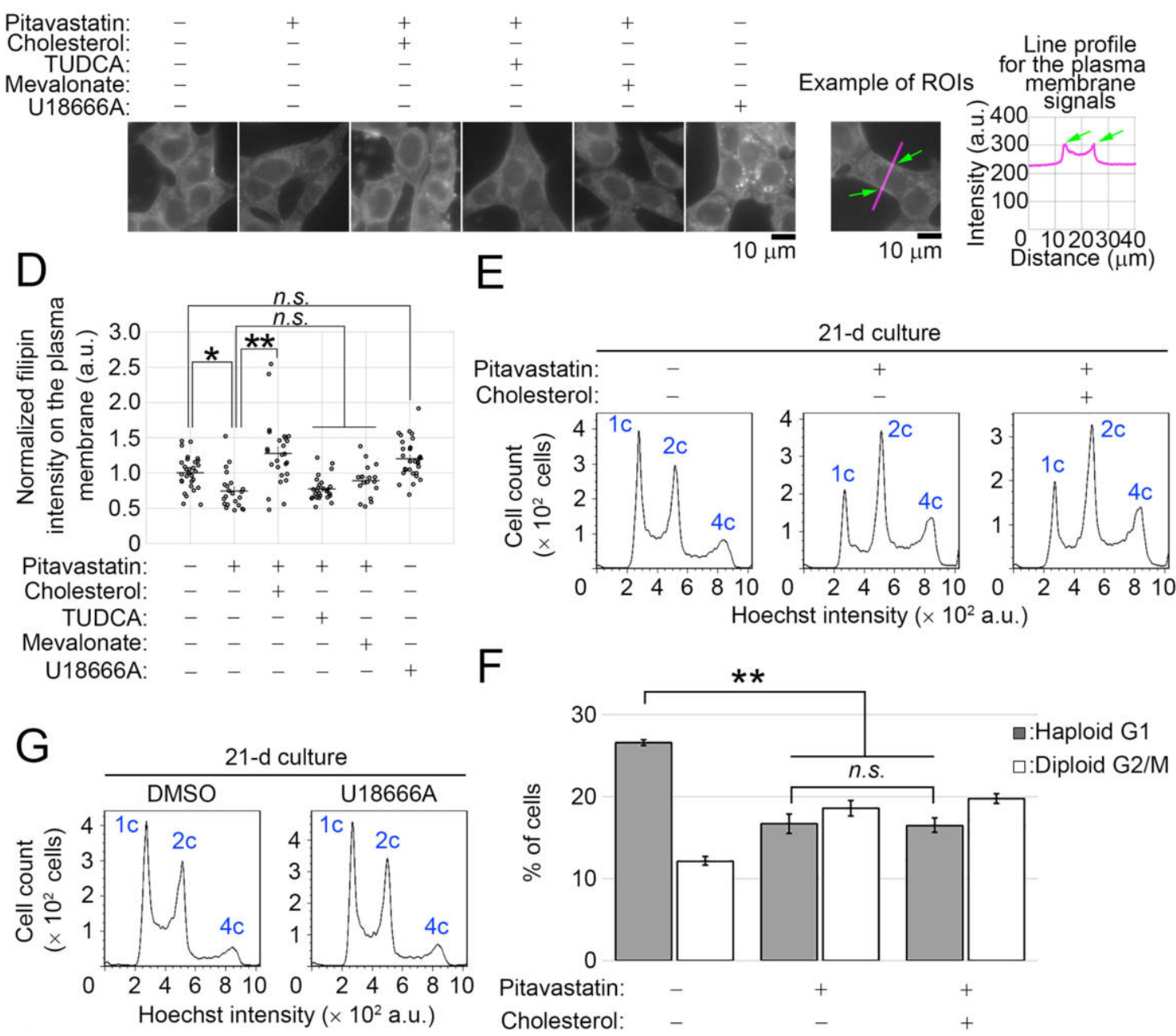

Fig. 2. Either supplementation or perturbation of cholesterol does not affect haploid stability. (A) Measurement of cholesterol extracted from the cells treated with the compounds for $1 \mathrm{~d}$. Means \pm SE of three independent experiments. There was no significant difference among these samples (one-way ANOVA with Tukey post-hoc test). (B) Fluorescence microscopy of HAP1 cells stained by filipin after treating with the compounds for $1 \mathrm{~d}$. (C, D) Quantification of filipin fluorescence intensity on the plasma membrane in B. The fluorescence signals on the plasma membrane were quantified from line profiles taken across the cells, as shown in C. Means $\pm \mathrm{SE}$ of at least 18 cells from two independent experiments $\left({ }^{*} p<0.05,{ }^{* *} p<0.01\right.$, one-way ANOVA with Tukey post-hoc test). (E) DNA content analysis after 21-d culture. Cells were cultured in the absence or presence of $0.5 \mu \mathrm{M}$ pitavastatin with or without $10 \mu \mathrm{M}$ cholesterol supplementation. (F) The proportion of the haploid G1 or diploid G2/M population in E. Means \pm SE of three independent experiments (day 21 in the long-term passages, ${ }^{* *} p<0.01$, one-way ANOVA with Tukey post-hoc test). (G) DNA content analysis after 21-d culture. Cells were cultured in the absence or presence of $2.5 \mu \mathrm{M} \mathrm{U}$ 18666A. Representative data from two independent experiments. 
A
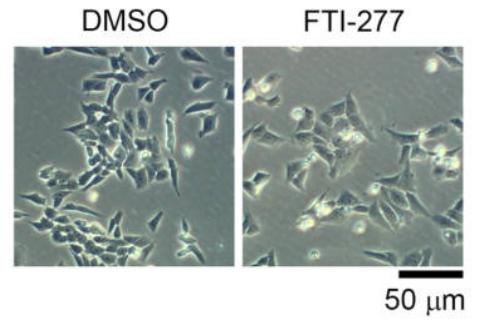

C

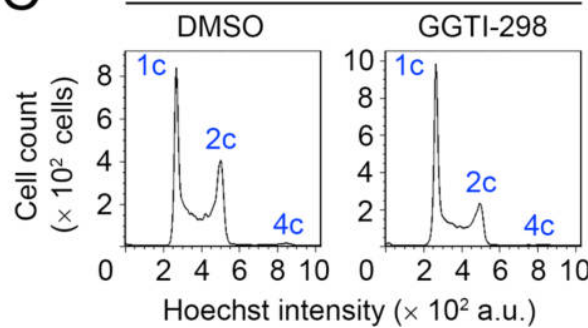

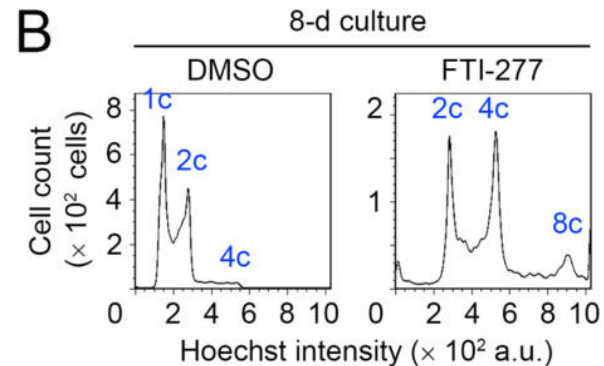

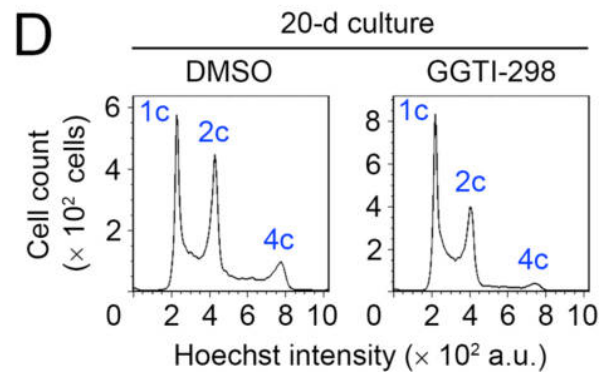

Fig. 3. Inhibition of protein prenylation does not phenocopy the statin-induced haploid destabilization. (A) Transparent microscopy of HAP1 cells treated with or without $20 \mu \mathrm{M}$ FTI-277 for $2 \mathrm{~d}$. Representative data from two independent experiments. (B-D) DNA content analysis after 8-d (B), 1-d (C), or 20d culture (D). Cells were cultured with or without $20 \mu \mathrm{M}$ FTI-277 (B) or $2 \mu \mathrm{M}$ GGTI-298 (C, D). Representative data from two independent experiments.

cal defect caused by the compound (Fig. 2B) (Reiners et al., 2011; Underwood et al., 1998). HAP1 cell proliferation was not severely affected by $2.5 \mu \mathrm{M}$ U18666A, allowing us to test its effect on the long-term haploid stability. In the long-term passages, DNA content profile was equivalent between non-treated control and $2.5 \mu \mathrm{M}$ U18666A-treated culture (Fig. 2G). This result further ruled out the possible involvement of cholesterol homeostatic control in haploid stability in HAP1 cells.

\section{Inhibition of protein prenylation does not phenocopy haploid destabilization by pitavastatin}

Among the mevalonate-derived metabolites, farnesyl pyrophosphate and geranylgeranyl pyrophosphate are used for the posttranslational prenylation of small GTPases that play crucial roles in the regulation of cell cycle and proliferation, as well as cell size control (Berndt et al., 2011; Miettinen and Björklund, 2015, 2016). Therefore, we tested the effects of FTI-277 or GGTI-298, which inhibits protein farnesylation or geranylgeranylation, respectively, on the stability of the haploid state in HAP1 cells. Twenty $\mu \mathrm{M}$ FTI-277 treatment caused mitotic progression defects marked by the round-shaped mitotically-arrested cells and abnormally enlarged cells in culture (Fig. 3A). Similar FTI-277-induced mitotic defects have been reported in different cell lines (Holland et al., 2015; Morgan et al., 2001; Moudgil et al., 2015). Consistent with the microscopic observation, FTI-277-treated HAP1 cells were drastically polyploidized within several days with the prominent accumulation of 2,4 , and $8 \mathrm{c}$ peaks in flow cytometric analysis
(Fig. 3B). This result suggests that FTI-277 induces wholegenome duplication in HAP1 cells regardless of the ploidy state, which was in contrast to the specific destabilization of haploid state by pitavastatin. The drastic polyploidization and subsequent cell death precluded us from testing the effects of FTI-277 on ploidy dynamics in a more extended period.

On the other hand, treatment with $2 \mu \mathrm{M}$ GGTI-298 mildly arrested haploid HAP1 cells at the G1 phase within $24 \mathrm{~h}$ (Fig. 3C), consistent with a previous report in several cell types (Sun et al., 1999). In prolonged culture for $20 \mathrm{~d}$ in the presence of $2 \mu \mathrm{M}$ GGTI-298, haploid G1 proportion was considerably conserved with smaller diploid G2/M proportion than non-treated control, presumably because of the moderate G1 arrest (Fig. 3D). Therefore, the suppression of either protein farnesylation or geranylgeranylation did not phenocopy the pitavastatin-induced haploid destabilization in our long-term experiment.

\section{Pitavastatin destabilizes the haploid state by evoking ER stress}

Since statins potentially induce ER stress by suppressing dolichol phosphates biosynthesis and inhibiting protein Nglycosylation (Chojnacki and Dallner, 1988), we next tested the possibility that pitavastatin destabilizes the haploid state through perturbing ER homeostasis. For this, we tested the effect of pitavastatin on ER stress in HAP1 cells using immunoblot analysis of several components in three signaling branches (i.e., the ATF6, IRE1 $\alpha$, and PERK pathways) of the unfolded protein response (UPR) (Kaufman, 
1999; Mori, 2000; Patil and Walter, 2001; Urano et al., 2000). We observed a consistent trend of a modest increase in the active cleaved form of ATF6 (Haze et al., 1999; Yoshida et al., 2000) and IRE1 $\alpha$ expression (Tsuru et al., 2016) upon 3-d treatment with $0.5 \mu \mathrm{M}$ pitavastatin treatment (Fig. 4A and B) in agreement with the previous studies in simvastatin-treated human cultured cells (Ghavami et al., 2014, 2012). The pitavastatin-treated cells also showed a trend of mild mobility shift of PERK in Phos-tag SDSPAGE gels (Fig. 4C), suggesting a mild increase in PERK phosphorylation (Harding et al., 1999). Consistent with this, pitavastatin treatment also resulted in a significant increase in the expression of ATF4 and CHOP, downstream components of the PERK pathway and multiple UPR branches, respectively (Fig. 4A and B) (Harding et al., 2000; Marciniak et al., 2004; Oyadomari and Mori, 2004). Mevalonate supplementation mostly canceled all of these changes in the UPR markers in pitavastatin-treated cells (Fig. 4A-C), demonstrating that pitavastatin evoked ER stress specifically through blocking mevalonate metabolism.

Finally, we determined whether ER stress induction is the cause of pitavastatin-mediated destabilization of haploid state in HAP1 cells. For this, we tested the effect of an ER stress-reducing chemical chaperone, tauroursodeoxycholic acid (TUDCA) (Ozcan et al., 2006; Yoon et al., 2016) on the haploid stability of HAP1 cells. Co-treatment with TUDCA did not affect ATF4 expression or PERK phosphorylation but substantially canceled the cleavage of ATF6 and the upregulation of IRE1 $\alpha$ and CHOP in pitavastatin-treated cells (Fig. 4A-C), presumably reflecting the complex effects of chemical chaperones on different factors in the UPR pathways (Uppala et al., 2017). In contrast, TUDCA did not change the cholesterol level in pitavastatin-treated cells (Fig. 2A-D). In long-term passages, co-treatment of TUDCA significantly preserved haploid G1 proportion in pitavastatin-treated cells (Fig. 4D and E). Therefore, restoration of ER homeostasis by TUDCA substantially improved the stability of the haploid state in the presence of pitavastatin, demonstrating that haploid destabilization by pitavastatin is caused, at least in part, through the induction of ER stress.

\section{Discussion}

It is assumed that ploidy differences have pleiotropic effects on intracellular biosynthetic processes and that the altered biosynthesis, in turn, affects cellular physiology at different ploidy states. However, it remains mostly elusive what biosynthetic processes have influences on ploidylinked cellular phenotypes. In this study, we found that an HMGCR inhibitor pitavastatin destabilized haploid state in human HAP1 cells. This result is consistent with the recent compound screen that identified statins to efficiently promote the expansion of diploidized population over haploids in HAP1 cell culture (Olbrich et al., 2019). As statins are widely used cholesterol-lowering drugs, the possible involvement of cholesterol metabolism in haploid stability has been suggested in the previous study (Olbrich et al., 2019). Interestingly, however, our results in the current study exclude this possibility for three reasons; 1) full restoration of cholesterol level by cholesterol supplementation did not improve haploid stability in statin-treated cells, 2) mevalonate supplementation fully restored haploid stability without restoring cholesterol level in statin-treated cells, and 3) cholesterol perturbation by a non-statin compound did not affect haploid stability.

Our data further specified the perturbation of mevalonate-mediated ER homeostatic control as a critical cause of the statin-induced haploid destabilization. Interestingly, pitavastatin-induced ER stress caused a drastic transition from haploid to diploid state without affecting the stability of diploid state in HAP1 cells. It remains unknown why the effect of pitavastatin on genome stability was specific to the haploid state. However, a possible reason might be a ploidy-dependent difference in tolerance to ER stress. Haploid cells are half in cell volume than diploid cells (Yaguchi et al., 2018), which presumably restricts intracellular spatial capacity for organelle structures. It has been demonstrated that the expansion of the ER lumen serves as a mechanism to increase ER capacity to ameliorate ER stress upon the accumulation of unfolded proteins (Bernales et al., 2006; Schuck et al., 2009; Shaffer et al., 2004; Sriburi et al., 2004). The lower availability of intracellular space may limit stress-responding ER expansion in haploid cells, hence lower tolerance to unfolded protein accumulation.

Mevalonate metabolism is an essential process that supports diverse biosynthetic pathways. Though we specified the preservation of ER homeostasis as a critical process underlying statin-mediate haploid destabilization, we cannot rule out other mevalonate-derived biosynthetic processes in haploid stability. For example, we cannot exclude the possibility that specific targets of protein farnesylation play roles in haploid stability, which might have been masked by the gross polyploidization upon the treatment with FTI-277. Comparative metabolome analysis would be a powerful approach to elucidate other biosynthetic processes playing critical roles in determining the physiology of cells at different ploidy states.

\footnotetext{
Acknowledgments. We thank the Global Facility Center at Hokkaido Univ for the flow cytometer. This work was supported by JSPS KAKENHI (Grant \#19J12210 to K.Ya., 19H03219, 19H05413, and 19KK0181 to R.U), the Hungarian National Research, Development and Innovation Office (NKFIH grant \#FK124442) and János Bolyai Research Scholarship from HAS to E.M., the Princess Takamatsu Cancer Research Fund, the Kato Memorial Bioscience Foundation, the Orange Foundation, the Smoking Research Foundation, the Suhara Memorial Foundation, and the Nakatani Foundation to R.U., and Bilateral Joint Research Projects of JSPS and HAS (JPJSBP120193801) to G.B., E.M., and R.U.
} 

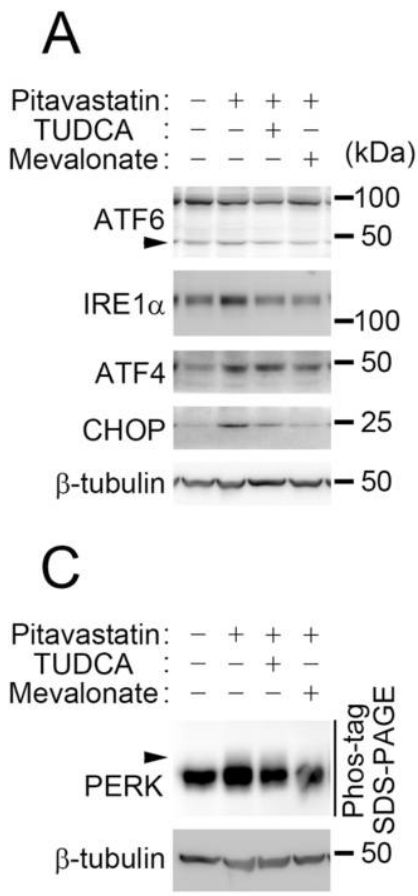

D
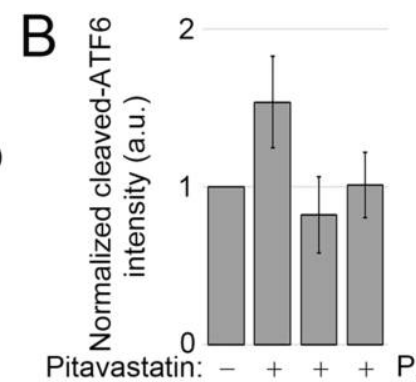

Pitavastatin: -+++

TUDCA: --+-

Mevalonate:
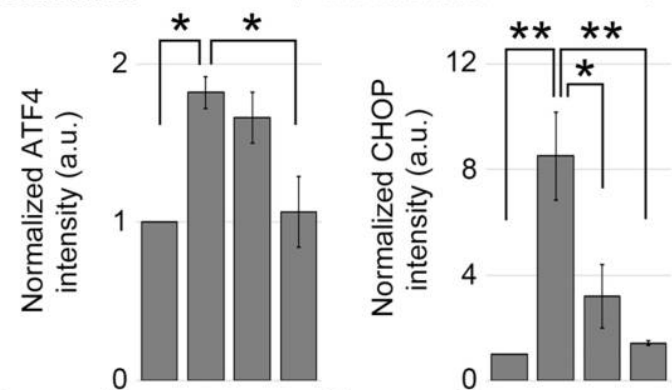

Pitavastatin

TUDCA:

Mevalonate:

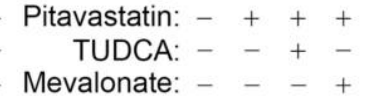

21-d culture

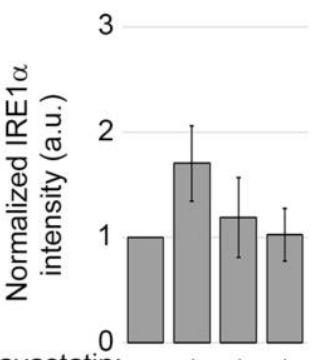

avastatin: -+++

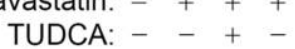

valonate: $-\quad-+$
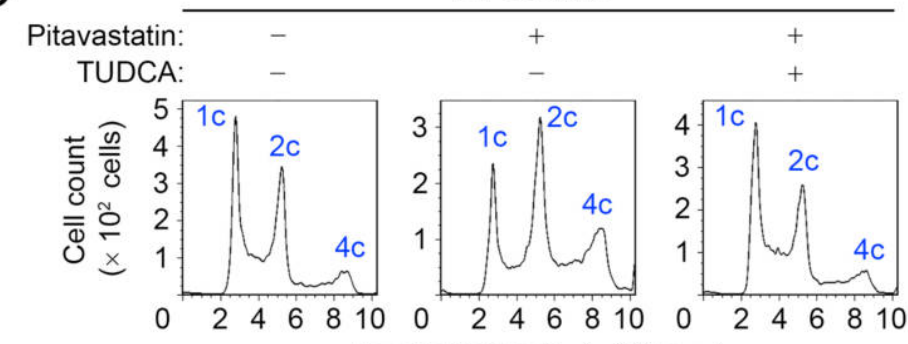

E

Hoechst intensity $\left(\times 10^{2}\right.$ a.u. $)$

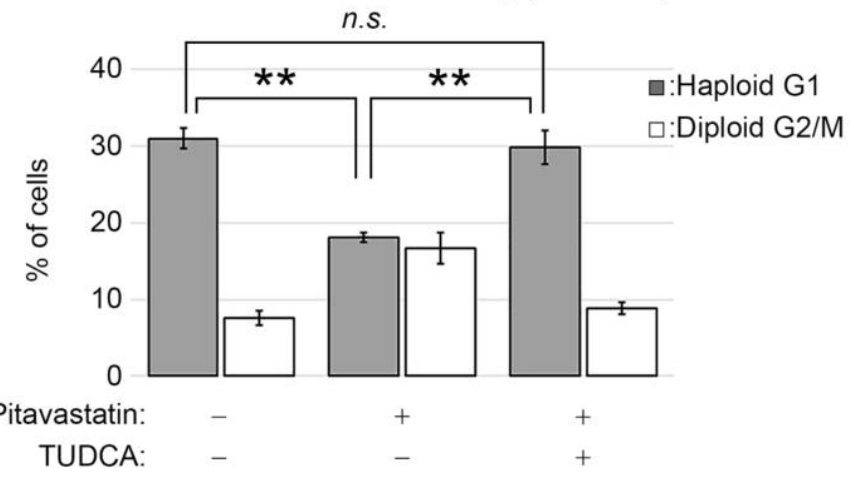

Fig. 4. Amelioration of ER stress improves haploid stability in pitavastatin-treated cells. (A, C) Immunoblotting of UPR components in HAP1 cells treated with the compounds for $3 \mathrm{~d}$. Cleaved ATF6 is indicated by the arrowhead. Immunoblotting of PERK was conducted using Phos-tag SDS-PAGE to detect phosphorylation-dependent band shift in $\mathrm{C}$ (the arrowhead). $\beta$-tubulin was detected as a loading control. Representative results from three independent experiments are shown. (B) Quantification of relative expression of UPR regulators/components in A. Means \pm SE of three independent experiments $\left({ }^{*} p<0.05,{ }^{*} p<0.01\right.$, one-way ANOVA with Tukey post-hoc test). (D) DNA content analysis after 21-d culture. Cells were cultured in the absence or presence of $0.5 \mu \mathrm{M}$ pitavastatin with or without $2.5 \mathrm{mM}$ TUDCA. (E) The proportion of the haploid G1 or diploid G2/M population in D. Means $\pm \mathrm{SE}$ of three independent experiments (day 21 in the long-term passages, ${ }^{* *} p<0.01$, one-way ANOVA with Tukey post-hoc test). 


\section{Author Contributions}

Conceptualization, K.Ya., and R.U.; Methodology, K.Ya., K. S., D.M., K.Yu., Y.I., G.B., E.M., and R.U.; Investigation, K.Ya, K.S. K.Yo., E.M., and R.U.; Formal Analysis, K.Ya., K.S., and R.U.; Resources, G.B., E.M., and R.U.; Writing-Original Draft, K.Ya., and R.U.; WritingReview \& Editing, K.Ya., D.M., K.Yu., E.M., and R.U.; Funding Acquisition, K.Ya., G.B., E.M., and R.U.

\section{References}

Adhyaru, B.B. and Jacobson, T.A. 2018. Safety and efficacy of statin therapy. Nat. Rev. Cardiol., 15: 757-769.

Bernales, S., McDonald, K.L., and Walter, P. 2006. Autophagy counterbalances endoplasmic reticulum expansion during the unfolded protein response. PLoS Biol., 4: e423.

Berndt, N., Hamilton, A.D., and Sebti, S.M. 2011. Targeting protein prenylation for cancer therapy. Nat. Rev. Cancer, 11: 775-791.

Buhaescu, I. and Izzedine, H. 2007. Mevalonate pathway: A review of clinical and therapeutical implications. Clin. Biochem., 40: 575-584.

Carette, J.E., Raaben, M., Wong, A.C., Herbert, A.S., Obernosterer, G., Mulherkar, N., Kuehne, A.I., Kranzusch, P.J., Griffin, A.M., Ruthel, G., Dal Cin, P., Dye, J.M., Whelan, S.P., Chandran, K., and Brummelkamp, T.R. 2011. Ebola virus entry requires the cholesterol transporter Niemann-Pick C1. Nature, 477: 340-343.

Carlberg, M., Dricu, A., Blegen, H., Wang, M., Hjertman, M., Zickert, P., Höög, A., and Larsson, O. 1996. Mevalonic Acid Is Limiting for NLinked Glycosylation and Translocation of the Insulin-like Growth Factor-1 Receptor to the Cell Surface: EVIDENCE FOR A NEW LINK BETWEEN 3-HYDROXY-3-METHYLGLUTARYL-COENZYME A REDUCTASE AND CELL GROWTH. J. Biol. Chem., 271: 1745317462.

Cenedella, R.J. 2009. Cholesterol synthesis inhibitor U18666A and the role of sterol metabolism and trafficking in numerous pathophysiological processes. Lipids, 44: 477-487.

Chojnacki, T. and Dallner, G. 1988. The biological role of dolichol. Biochem. J., 251: 1-9.

Doucey, M.A., Hess, D., Cacan, R., and Hofsteenge, J. 1998. Protein Cmannosylation is enzyme-catalysed and uses dolichyl-phosphatemannose as a precursor. Mol. Biol. Cell, 9: 291-300.

Drabikowski, W., Lagwińska, E., and Sarzala, M.G. 1973. Filipin as a fluorescent probe for the location of cholesterol in the membranes of fragmented sarcoplasmic reticulum. Biochim. Biophys. Acta, 291: 61-70.

Ghavami, S., Yeganeh, B., Stelmack, G.L., Kashani, H.H., Sharma, P., Cunnington, R., Rattan, S., Bathe, K., Klonisch, T., Dixon, I.M., Freed, D.H., and Halayko, A.J. 2012. Apoptosis, autophagy and ER stress in mevalonate cascade inhibition-induced cell death of human atrial fibroblasts. Cell Death Dis., 3: e330.

Ghavami, S., Sharma, P., Yeganeh, B., Ojo, O.O., Jha, A., Mutawe, M.M., Kashani, H.H., Los, M.J., Klonisch, T., Unruh, H., and Halayko, A.J. 2014. Airway mesenchymal cell death by mevalonate cascade inhibition: integration of autophagy, unfolded protein response and apoptosis focusing on Bcl2 family proteins. Biochim. Biophys. Acta, 1843: 12591271.

Harding, H.P., Zhang, Y., and Ron, D. 1999. Protein translation and folding are coupled by an endoplasmic-reticulum-resident kinase. Nature, 397: 271-274.

Harding, H.P., Novoa, I., Zhang, Y., Zeng, H., Wek, R., Schapira, M., and Ron, D. 2000. Regulated translation initiation controls stress-induced gene expression in mammalian cells. Mol. Cell, 6: 1099-1108.

Haze, K., Yoshida, H., Yanagi, H., Yura, T., and Mori, K. 1999. Mamma- lian transcription factor ATF6 is synthesized as a transmembrane protein and activated by proteolysis in response to endoplasmic reticulum stress. Mol. Biol. Cell, 10: 3787-3799.

Holland, A.J., Reis, R.M., Niessen, S., Pereira, C., Andres, D.A., Spielmann, H.P., Cleveland, D.W., Desai, A., and Gassmann, R. 2015. Preventing farnesylation of the dynein adaptor Spindly contributes to the mitotic defects caused by farnesyltransferase inhibitors. Mol. Biol. Cell, 26: 1845-1856.

Kaufman, R.J. 1999. Stress signaling from the lumen of the endoplasmic reticulum: coordination of gene transcriptional and translational controls. Genes Dev., 13: 1211-1233.

Leung, K.F., Baron, R., and Seabra, M.C. 2006. Thematic review series: lipid posttranslational modifications. geranylgeranylation of Rab GTPases. J. Lipid Res., 47: 467-475.

Marciniak, S.J., Yun, C.Y., Oyadomari, S., Novoa, I., Zhang, Y., Jungreis, R., Nagata, K., Harding, H.P., and Ron, D. 2004. CHOP induces death by promoting protein synthesis and oxidation in the stressed endoplasmic reticulum. Genes Dev., 18: 3066-3077.

Miettinen, Teemu P., Pessa, Heli K.J., Caldez, Matias J., Fuhrer, T., Diril, M.K., Sauer, U., Kaldis, P., and Björklund, M. 2014. Identification of Transcriptional and Metabolic Programs Related to Mammalian Cell Size. Curr. Biol., 24: 598-608.

Miettinen, T.P. and Björklund, M. 2015. Mevalonate Pathway Regulates Cell Size Homeostasis and Proteostasis through Autophagy. Cell Reports, 13: 2610-2620.

Miettinen, T.P. and Björklund, M. 2016. Cellular Allometry of Mitochondrial Functionality Establishes the Optimal Cell Size. Dev. Cell, 39: 370-382.

Morgan, M.A., Dolp, O., and Reuter, C.W.M. 2001. Cell-cycle-dependent activation of mitogen-activated protein kinase kinase (MEK-1/2) in myeloid leukemia cell lines and induction of growth inhibition and apoptosis by inhibitors of RAS signaling. Blood, 97: 1823-1834.

Mori, K. 2000. Tripartite management of unfolded proteins in the endoplasmic reticulum. Cell, 101: 451-454.

Moudgil, D.K., Westcott, N., Famulski, J.K., Patel, K., Macdonald, D., Hang, H., and Chan, G.K. 2015. A novel role of farnesylation in targeting a mitotic checkpoint protein, human Spindly, to kinetochores. $J$. Cell Biol., 208: 881-896.

Mullen, P.J., Yu, R., Longo, J., Archer, M.C., and Penn, L.Z. 2016. The interplay between cell signalling and the mevalonate pathway in cancer. Nat. Rev. Cancer, 16: 718-731.

Olbrich, T., Vega-Sendino, M., Murga, M., de Carcer, G., Malumbres, M., Ortega, S., Ruiz, S., and Fernandez-Capetillo, O. 2019. A Chemical Screen Identifies Compounds Capable of Selecting for Haploidy in Mammalian Cells. Cell Reports, 28: 597-604.e594.

Oyadomari, S. and Mori, M. 2004. Roles of CHOP/GADD153 in endoplasmic reticulum stress. Cell Death Differ., 11: 381-389.

Ozcan, U., Yilmaz, E., Ozcan, L., Furuhashi, M., Vaillancourt, E., Smith, R.O., Görgün, C.Z., and Hotamisligil, G.S. 2006. Chemical chaperones reduce ER stress and restore glucose homeostasis in a mouse model of type 2 diabetes. Science, 313: 1137-1140.

Patil, C. and Walter, P. 2001. Intracellular signaling from the endoplasmic reticulum to the nucleus: the unfolded protein response in yeast and mammals. Curr. Opin. Cell Biol., 13: 349-355.

Reiners, J.J., Jr., Kleinman, M., Kessel, D., Mathieu, P.A., and Caruso, J.A. 2011. Nonesterified cholesterol content of lysosomes modulates susceptibility to oxidant-induced permeabilization. Free Radic. Biol. Med., 50: 281-294.

Sagi, I. and Benvenisty, N. 2017. Haploidy in Humans: An Evolutionary and Developmental Perspective. Dev. Cell, 41: 581-589.

Schuck, S., Prinz, W.A., Thorn, K.S., Voss, C., and Walter, P. 2009. Membrane expansion alleviates endoplasmic reticulum stress independently of the unfolded protein response. J. Cell Biol., 187: 525-536. 
Shaffer, A.L., Shapiro-Shelef, M., Iwakoshi, N.N., Lee, A.-H., Qian, S.-B., Zhao, H., Yu, X., Yang, L., Tan, B.K., Rosenwald, A., Hurt, E.M., Petroulakis, E., Sonenberg, N., Yewdell, J.W., Calame, K., Glimcher, L.H., and Staudt, L.M. 2004. XBP1, Downstream of Blimp-1, Expands the Secretory Apparatus and Other Organelles, and Increases Protein Synthesis in Plasma Cell Differentiation. Immunity, 21: 81-93.

Sriburi, R., Jackowski, S., Mori, K., and Brewer, J.W. 2004. XBP1: a link between the unfolded protein response, lipid biosynthesis, and biogenesis of the endoplasmic reticulum. J. Cell Biol., 167: 35-41.

Sun, J., Qian, Y., Chen, Z., Marfurt, J., Hamilton, A.D., and Sebti, S.M. 1999. The geranylgeranyltransferase I inhibitor GGTI-298 induces hypophosphorylation of retinoblastoma and partner switching of cyclindependent kinase inhibitors. A potential mechanism for GGTI-298 antitumor activity. J. Biol. Chem., 274: 6930-6934.

Tsuru, A., Imai, Y., Saito, M., and Kohno, K. 2016. Novel mechanism of enhancing IRE1 $\alpha$-XBP1 signalling via the PERK-ATF4 pathway. Sci. Rep., 6: 24217.

Underwood, K.W., Jacobs, N.L., Howley, A., and Liscum, L. 1998. Evidence for a cholesterol transport pathway from lysosomes to endoplasmic reticulum that is independent of the plasma membrane. J. Biol. Chem., 273: 4266-4274.

Uppala, J.K., Gani, A.R., and Ramaiah, K.V.A. 2017. Chemical chaperone, TUDCA unlike PBA, mitigates protein aggregation efficiently and resists ER and non-ER stress induced HepG2 cell death. Sci. Rep., 7:
3831

Urano, F., Bertolotti, A., and Ron, D. 2000. IRE1 and efferent signaling from the endoplasmic reticulum. J. Cell Sci., 113 Pt 21: 3697-3702.

Wang, M. and Casey, P.J. 2016. Protein prenylation: unique fats make their mark on biology. Nat. Rev. Mol. Cell Biol., 17: 110-122.

Wutz, A. 2014. Haploid animal cells. Development, 141: 1423-1426.

Yaguchi, K., Yamamoto, T., Matsui, R., Tsukada, Y., Shibanuma, A., Kamimura, K., Koda, T., and Uehara, R. 2018. Uncoordinated centrosome cycle underlies the instability of non-diploid somatic cells in mammals. J. Cell Biol., 217: 2463-2483.

Yang, L., Xue, Z., He, Y., Sun, S., Chen, H., and Qi, L. 2010. A Phos-tagbased approach reveals the extent of physiological endoplasmic reticulum stress. PLoS One, 5: e11621-e11621.

Yoon, Y.M., Lee, J.H., Yun, S.P., Han, Y.S., Yun, C.W., Lee, H.J., Noh, H., Lee, S.J., Han, H.J., and Lee, S.H. 2016. Tauroursodeoxycholic acid reduces ER stress by regulating of Akt-dependent cellular prion protein. Sci. Rep., 6: 39838

Yoshida, H., Okada, T., Haze, K., Yanagi, H., Yura, T., Negishi, M., and Mori, K. 2000. ATF6 activated by proteolysis binds in the presence of NF-Y (CBF) directly to the cis-acting element responsible for the mammalian unfolded protein response. Mol. Cell. Biol., 20: 6755-6767.

(Received for publication, November 5, 2020, accepted, December 12, 2020 and published online, December 22, 2020) 